\title{
The development of the Monthly Business Survey
}

Craig Taylor, Gareth James and Peter Pring

Office for National Statistics

\section{Summary}

The Monthly Business Survey (MBS) brings together the Office for National Statistics' (ONS) short term surveys on different sectors of the economy. The Monthly Production Inquiry and the Monthly Inquiry into the Distribution and Services Sector have been integrated into one survey. Surveys of the construction sector remain separate but have been redesigned in a similar way to MBS. The Retail Sales Inquiry has also remained separate, but by rebranding as the MBS it paves the way for future integration. By standardising survey deigns and harmonising methodologies ONS hopes to achieve a number of benefits. This article reports on the development of the MBS to date - including the scope of the survey and various aspects of the survey redesign.

\section{Introduction}

The Office for National Statistics (ONS) launched the Monthly Business Survey (MBS) in January 2010, bringing together short term surveys that cover different sectors of the economy. The quarterly employment only survey (formally known as Gaps) was also re-branded as the Quarterly Business Survey (QBS) and has been extended to cover all production and service industries. The introduction of MBS had close links with a number of other projects that were conducted internally within ONS. These included the Standard Industrial Classification (SIC) 2007 implementation project, Telephone Data Entry (TDE) project, Workforce Jobs Redevelopment (Barford 2010), Construction Statistics (Sharp 2010) and the Eden project on selective editing. It was recognised that the development work associated with the implementation of MBS could be achieved by using resources from these other projects. Therefore, ONS took the opportunity of delivering the benefits associated with the introduction of MBS at the same time as delivering these existing projects.

Information on the implementation of Standard Industrial Classification 2007 (SIC 2007) has been provided in other articles (Hughes 2009; Hughes 2010). A new sample design with strata based on SIC 2007 codes, was implemented for many short term business surveys at the start of 2010.

Although a change in SIC does not necessarily prompt a survey redesign (results on the new SIC 
can still be derived from a survey stratified on the old SIC), ONS has reviewed the design and implemented a new one for each of the surveys. In the case of the Monthly Production Inquiry (MPI) and the Monthly Inquiry into the Distribution and Services Sector (MIDSS), ONS decided to go one stage further and integrate these surveys to produce the MBS. This paper focuses on what this development work included. Although MPI and MIDSS were the only two surveys that were fully integrated, information on other surveys that fell into scope of the developments has been provided in this article.

\section{Background - short term surveys prior to January 2010}

Prior to January 2010, ONS conducted a number of short term surveys that had a monthly or quarterly periodicity. Although these surveys generally covered different parts of the economy, the information on the questionnaires collected was similar. Whilst trying to deliver genuine improvements within resources of existing projects, it was important to remain pragmatic about what could be achieved in a short space of time. Therefore, the initial stages of the MBS project focused on only a few specified short term surveys. However, the project was set up in such a way that it will be possible to extend MBS to encompass other surveys in the future.

The surveys under consideration for the initial phase of the MBS project were:

- Monthly Inquiry into the Production Industries (MPI). A monthly survey of 8,000 businesses in the production sector. It collected total turnover, export turnover and employment information, as well as a few other variables (such as value of orders) in some SICs. A few industries received an employment-only (where the collection of turnover was not required) MPI questionnaire, as other sources of information were used to obtain turnover/output information for National Accounts. Turnover estimates form the main input to the Index of Production (IoP), itself used in the output measure of Gross Domestic Product (GDP). Employment data are used to compile Workforce Jobs.

- Monthly Inquiry into the Distribution and Services Sector (MIDSS). A monthly survey of 25,000 businesses in the distribution and other services sectors. It collected information on turnover on a monthly basis from the entire sample, and employment information from a sub-sample every third month (to give quarterly estimates). Turnover estimates form the main input to the Index of Services (loS), itself used in GDP. Employment data are used to compile the estimate of Workforce Jobs.

- In contrast to the production sector, a separate survey was run on a quarterly basis to collect employment information from businesses in the service sector where the collection of turnover data was not required. The survey was commonly known at ONS as Gaps. The data were used to compile the estimate of Workforce Jobs.

- Retail Sales Inquiry (RSI). A survey of 5,000 businesses in the retail sector, where the RSI 'month' refers to either a four- or five-week period. It collects total retail sales (as opposed to total turnover) each period; and employment questions were asked to the whole sample every third period (that is quarterly). Sales estimates are the main input to the Retail Sales Index; employment data are used to compile the estimate of Workforce Jobs. 
- Construction statistics. New surveys were being developed by ONS to cover the construction industry; the industry was previously surveyed by another government department. Data were to be published in their own right, as well as forming part of the output measure of GDP.

Each of the surveys listed had developed independently. Therefore, each survey had its own design, and differences existed in the details of the methodology. However, the same processing software was used for each survey, and the underlying aims and outputs were all similar. Thus, it was seen that large benefits could be obtained by standardising the designs and harmonising the methodology.

\section{What has been achieved - survey redesign}

The main outcome of the MBS project has been that MPI and MIDSS were merged into one survey from January 2010. This includes the data collection right through to the operational processing of the survey - meaning only one database instead of two separate databases is now used. Due to the operational difficulty of combining different periodicity (four or five week periods instead of a calendar month) into a single survey, RSI has remained separate. However, the RSI questionnaires are now headed 'Monthly Business Survey', paving the way for future integration. The construction surveys also remain separate, but have been redesigned in a similar way to MBS. The Quarterly Business Survey (QBS) is now the name of the survey that collects employment information only, and its scope includes industries from both the production sector and the services sector.

\section{Scope of the survey}

One of the most important and lengthy tasks of the redesign was to first agree upon the scope of the surveys, in terms of which SIC 2007 codes would be included. It was then important to agree upon the SIC code aggregations that would be used for publication of outputs, which in turn would form the sampling strata in most cases. The views of the main internal customers were key (such as National Accounts), and external customers were consulted via the output managers.

When deciding the scope of MBS, the predecessor surveys (MPI and MIDSS) provided a good initial basis for decisions on strata where industries that existed separately under SIC 2003 continued to do so under SIC 2007. However, there were some new industries to consider for inclusion. For example landscape gardening moved from Agriculture (previously out of scope) to Services. Other industries that were more or less out of scope under SIC 2003 before but contained moderate numbers of businesses in scope under SIC 2007 were also carefully considered. In addition, there had been a long-standing desire from key customers to bring into our short term surveys some industries (such as veterinary activities) for which data had previously been sourced from elsewhere, but the direct collection of turnover would be preferred. Some of these SICs were included in the MBS sample from January 2010. Other SICs were introduced in 
July 2010 following cognitive testing of the questions by the data collection methodology team. This ensured that collection of data from businesses in these previously unsampled industries were feasible.

A final influencing factor was to reduce some of the detail that existed. There had previously been too many strata (especially in the production sector) to allow a truly optimal design for the limited sample size available. Accommodating possibly smaller sample sizes also increased the desire for fewer strata. For RSI, the number of SIC strata has stayed approximately the same at 27 . For the collection of both turnover and employment, just over 300 SIC strata in MPI and MIDSS have been reduced to approximately 150 in MBS. For the employment-only industries, about 40 in MPI and Gaps have been reduced to approximately 30 in QBS.

\section{Survey redesign}

The development work associated with the survey redesign can be separated into a number of sub-categories along the Statistical Value Chain (SVC).

\section{Questionnaire design}

One of the overall aims of the redesign was to present a single, standardised survey to respondents. Since the project did not fully integrate all short term surveys, a decision was taken to standardise the survey name for a number of short term surveys as a minimum. The rationale behind the decision to remove the activity of the contributor in the title of the survey was:

- there were a few oddities with the names of the short term surveys prior to January 2010. For example, RSI was sent out under the banner of MIDSS

- a number of SICs moved between services, manufacturing, retail and construction as a result of moving towards SIC 2007. It was felt that it could be potentially confusing for respondents who are sent a different questionnaire as a result of the change, for example MPI instead of MIDSS. Therefore, it was felt that sending out all short term surveys under a MBS banner may help the transition period; and

- the potential of expanding MBS in future years. Since all the proposals for the initial MBS project needed to be achieved within the resource of existing projects, a pragmatic approach to the scope was taken. Therefore, it was agreed that only MPI and MIDSS would be truly integrated. However, as the vision for the office is to reduce the number of our surveys, renaming all short term surveys was seen as paving a way for this future development.

It was agreed that the title of the surveys would become the Monthly Business Survey, or Quarterly Business Survey for those SICs that asked only employment questions on a quarterly basis. This name change occurred at a similar time to the name change of the ONS's structural business survey, which was renamed as the Annual Business Survey. These name changes have provided some consistency to the branding of our business surveys. 
A separate review and consultation about employment statistics had concluded that employment data only needed to be collected quarterly, a change for the production sector in which it had previously been collected monthly on MPI. Only a sub-sample of businesses in MIDSS were also asked the employment questions, and this practice has been retained on MBS and also extended to RSI. This approach reduces burden on businesses, but still produces employment estimates of acceptable quality (employment data tend to be less variable than turnover data, especially since strata sizebands are defined by employment).

There has been a large reduction in the number of different questionnaire types for MBS compared to the combined total for MPI and MIDSS. This is largely the result of a Triennial Review conducted by the Short Term Turnover Inquiries (STTI) team, who run the MBS and formally MPI and MIDSS. The review identified several questions that were redundant following consultation with users. The reduction in the number of distinct questionnaire types has enabled the Telephone Date Entry project to be rolled out in a more simplified manner. There are now 26 different questionnaire types covering all MBS industries, compared with the 65 questionnaire types that were previously used for MPI and MIDSS.

\section{Sample design}

Each of the short term surveys were stratified as a cross-classification of SIC and employment sizeband. For MPI, RSI, MIDSS and Gaps, four employment sizebands were used in each SIC with the largest businesses being completely enumerated. In RSI and MIDSS there was an additional band comprising businesses in the middle two employment sizebands, but with turnover exceeding a given threshold (these businesses were also completely enumerated). Different sets of sizeband boundaries were used in each survey, and in some surveys there was more than one set of sizebands in use.

This meant there was no one obvious set of sizebands to use for MBS. Although operationally more convenient, it soon became apparent that just one set of sizebands would not be appropriate for use throughout MBS. As an example, the largest businesses in production sector industries tend to be smaller than the largest ones in the services sector. Therefore, it was decided that a limited number of sets of sizebands would be used, and that the most appropriate set of sizebands would be chosen on an industry-by-industry basis.

Different methods for choosing the stratum sizebands boundaries were tested on both turnover and employment data. After determining 'optimal' boundaries within each SIC industry stratum, a compromise was reached which best served both turnover and employment, subject to the boundaries coming from a limited set of options. In contrast to having several sizebands within MBS, the same set of sizebands was used for all SIC strata in RSI. However, these have changed from the sizebands under SIC 2003 following investigations that a revision to the boundaries would give a more efficient sample. In RSI, previous 0-9, 10-19 and 20-99 bands were replaced with 04, 5-9 and 10-99, with complete enumeration of 100+ employment businesses remaining the same.

ONS operates a policy of limiting the burden on businesses with under 10 employment, by imposing a maximum time in sample of 15 months or five quarters. Although it has traditionally 
been the case that a $0-9$ sizeband has been used, investigations into the mechanics of sample selection and rotation revealed that this policy could still be applied, even with a 5-19 size band that straddles the threshold on burden applied in MBS industries. Inconsistencies in sample rotation rates between the production and services sectors have been removed.

\section{Sample allocation}

The main principle for allocating a sample in ONS business surveys is to follow Neyman optimal allocation, for which the population size and standard deviation of the data in each stratum must be known or estimated. The population sizes are easily obtained from the business register, but getting a robust estimate of the standard deviation is a more challenging operation. To ensure a robust allocation, weighted standard deviations for the new strata were calculated from data collected on the old designs over a period of 12 recent months, and then averaged. In the cases of SICs that were new to the survey, modelled estimates of standard deviations were used, and the allocations will be reviewed in the future in light of the data collected.

The sample was allocated in a way that not only aimed to give good precision at the overall level of aggregation, but also met target values for the coefficient of variation at various sub-aggregates levels, usually the main groups used for publication. There has been no change in the overall sample size, but the sample has been allocated across the combined production and services sectors. Previously, these had always been considered separately when reviewing the samples with fixed sample sizes in each sector.

\section{Data editing and imputation}

Editing rules are used to validate data returned by respondents. Any rules used that were SICspecific were reviewed and changed to accommodate SIC 2007. The opportunity was also taken to remove any redundant rules. A new strategy for editing is also being introduced at ONS, and will be applied to the short term surveys in the near future. Changes made to the procedures on the introduction of MBS were applied in a way that would allow future changes to be applied more easily. Indeed, the Eden principles of selective editing were successfully implemented in RSI in July 2010 and MBS in August 2010.

In cases where a business fails to respond to the survey, values are imputed based upon information that is available. In most cases ratio imputation is used, based upon information from responding businesses in the same imputation class in the same period. This approach minimises the potential for bias from differential non-response. However, MPI and MIDSS had different ways of forming imputation classes. MPI used an industry-based approach to imputation, while MIDSS used a sampling-stratum-based approach. The processing systems were constrained such that only one method of imputation could be applied to the whole survey, and so a decision was required for MBS - that is whether to follow MPI practice or MIDSS practice (or to do something else). Testing was carried out to determine the effect on historical data of switching either survey to the other method of imputation, and the conclusion was that the MPI approach was better and 
would be used in the new MBS. It was both more robust and less prone to error when compared to the MIDSS approach.

There were also a number of smaller changes required to the editing process to enable MPI and MIDSS to be integrated. For example, the treatment of partial non-response and the date adjustments methodology was inconsistent. In both cases an investigation was undertaken and a consistent approach was taken for MBS following methodological advice.

\section{Estimation}

The surveys use ratio estimation for both turnover and employment, calibrating to known population totals on the business register. The estimator of turnover (and similar variables) uses register turnover as the auxiliary variable and employment type variables use register employment. However, whereas MIDSS used separate ratio estimation with calibration taking place within each sampling stratum, MPI used combined ratio estimation where the non-completely enumerated sizebands within each SIC stratum were considered as one for calibration. RSI had a mix of these two methods, with some SICs using separate ratio estimation and some combined ratio estimation.

It was decided to apply the best estimator for each SIC on a case-by-case basis for MBS - that is deciding whether to use separate or combined ratio estimation based upon the characteristics of each SIC. Following general advice, the default position was to use separate ratio estimation. However, where there was a good reason to use combined ratio estimation (mainly small sample sizes and no obvious difference in the model parameter estimate between the size bands), then this method was chosen. Empirical investigations were used to decide on the estimator to be used in each case.

\section{Changes to the processing system}

An off-line test version of the processing system was configured. This was based on the combination of the sample design and allocation, choice of estimator, questionnaire type and changes to the editing and imputation rules. This meant that full testing could be carried out in advance of the test system becoming the live system from January 2010.

In terms of practicality, MPI and MIDSS were merged into one database (processing system). Whereas the production and services parts of the system were previously operated separately, they would now operate as one, although the teams that operated the former MPI and MIDSS were already located in the same division. Combining the surveys necessitated a number of changes in operation, and opportunities were taken to eliminate inconsistencies in timing and methods of delivery of turnover data to National Accounts.

Some of the changes in operation that have led to a consistent approach to SICs in production and services are:

- a harmonised approach has been adopted to a number of results processing functions. This includes the generation of a Scottish Government extract, the generation of data to inform early 
estimates of GDP (this was only previously carried out on the services SICs) and a consistent approach to revising results across all MBS SICs

- a harmonised delivery has been established to the Economic Labour and Social Analysis Directorate for production and services; and

- a new briefing strategy covering both growth and revisions has been introduced and adapted for SIC 2007 introducing consistency across production and services industries

These changes removed inconsistencies and paved the way for the MPI and MIDSS surveys to be processed as MBS.

\section{Publications}

A new publication called Turnover, Orders in Production and Services Industries (TOPSI) was introduced for January 2010 data, first published in March 2010. This is published at a mixture of two and three digit SIC 2007 industry levels. TOPSI integrated and replaced the Engineering, Turnover and Orders Digest (ETOD) and Distribution of Services Turnover (DST) release, making several extensions to their industry coverage:

- UK turnover figures have been extended beyond 'Engineering' to include other 'Production' industries such as 'Mining and quarrying', 'Manufacture of food products' and 'Manufacture of rubber and plastic products'

- Export turnover has been extended to cover most of 'Manufacturing'

- Turnover figures for 'Services' (formerly called 'Distribution and Services') are still on a Great Britain (GB) basis and now include 'Publishing

- New orders figures previously only published for 'Engineering' have also been extended to cover other 'Manufacturing' industries such as 'Manufacture of textiles' and 'Manufacture of chemicals and chemical products'. The Orders on hand figures are no longer published

Continuous SIC 2007 time series on a consistent basis are required to maintain comparability of estimates over time. To ensure this, the following approaches were used for individual time series:

- for historical data from January 1998 to December 2008 a conversion matrix has been used. This method apportions industry-based estimates for businesses under SIC 2003 and then reaggregates the estimates to form SIC 2007 estimates. The proportions used are obtained from dual-coded data on the ONS business register. This method was used by the ONS at the last major change in classification and is widely used by other National Statistics Institutes

- for data from January 2009 to December 2009, micro-data (individual survey responses) have been re-weighted and aggregated to form the estimates for SIC 2007 based domains. The results using this domain estimation method have been calibrated to the appropriate population totals. This is a standard statistical method, which is expected to give improved estimates compared to the use of conversion matrices, and to match closely to estimates derived from the actual survey data 
- from January 2010 onwards, survey estimates are calculated using data obtained from the redesigned sample. This is the standard survey approach and follows previously used statistical methods.

It is important to remove differences based on method changes as any changes between estimates need to reflect real world changes rather than changes in methodology. To ensure consistent estimates over time, any differences between the converted and domain estimates at January 2009 are taken into account through a linking process. This ensures there is no discontinuity between the data produced using the conversion matrix and domain estimation methods. The movements at the current end of the series reflect the new sample design from January 2010 onwards and have been checked against December 2009 data for possible discontinuities.

\section{Contact}

Craig.taylor@ons.gsi.gov.uk

\section{References}

Barford N (2010) 'Revisions to Workforce Jobs', Economic \& Labour Market Review, vol 9, no 9, pp 73-79

Sharp G and Crook T (2010) 'Development of Construction Statistics', Economic \& Labour Market Review, vol 4, no 3, pp 52-58

Hughes J C and Brook K (2009) 'Implementation of SIC 2007 across the Government Statistical Service', Economic \& Labour Market Review, vol 3, no 8, pp 67-69

Hughes J C, James G and Evans E (2009) 'Implementation of Standard Industrial Classification 2007: December 2009 update', Economic \& Labour Market Review, vol 3, no 12, pp 51-55 